\title{
Significant differences in flow standardised breath sound spectra in patients with chronic obstructive pulmonary disease, stable asthma, and healthy lungs
}

\author{
L Pekka Malmberg, Leena Pesu, Anssi R A Sovijärvi
}

\begin{abstract}
Background - Spectral characteristics of breath sounds in asthma and chronic obstructive pulmonary disease (COPD) have not previously been compared, although the structural differences in these disorders might be reflected in breath sounds. Methods - Flow standardised inspiratory breath sounds in patients with COPD $(n=$ 17) and stable asthma $(n=10)$ with significant airways obstruction and in control patients without any respiratory disorders $(n=11)$ were compared in terms of estimates of the power spectrum. Breath sounds were recorded simultaneously at the chest and at the trachea.

Results - The median frequency $\left(F_{50}\right)$ of the mean (SD) breath sound spectra recorded at the chest was higher in asthmatics (239 (19) $\mathrm{Hz}$ ) than in both the control patients $(206(14) \mathrm{Hz})$ and the patients with COPD (201 (21) Hz). The total spectral power of breath sounds recorded at the chest in terms of root mean square (RMS) was higher in asthmatics than in patients with COPD. In patients with COPD the spectral parameters were not statistically different from those of control patients. The $F_{50}$ recorded at the trachea in the asthmatics was significantly related to forced expiratory volume in one second $\left(F_{E V}\right)(r=-0 \cdot 77)$, but this was not seen in the other groups.

Conclusions - The observed differences in frequency content of breath sounds in patients with asthma and COPD may reflect altered sound generation or transmission due to structural changes of the bronchi and the surrounding lung tissue in these diseases. Spectral analysis of breath sounds may provide a new non-invasive method for differential diagnosis of obstructive pulmonary diseases.

(Thorax 1995;50:1285-1291)
\end{abstract}

Keywords: respiratory sounds, asthma, chronic obstructive pulmonary disease.

Computer assisted techniques for lung sound analysis $^{1-6}$ have provided new data on the relationship between respiratory sounds and different pathophysiological conditions of the lung. By using these methods, changes in pulmonary and tracheal breath sounds as well as adventitious breath sounds - for example, wheezes and crackles - can be objectively and accurately recorded. In early studies Forgacs ${ }^{7}$ showed that breath sound intensity at the mouth was associated with forced expiratory volume in one second $\left(\mathrm{FEV}_{1}\right)$ in patients with asthma and chronic obstructive bronchitis, but not in those with emphysema. Later it was shown that lung sound intensity is related to regional distribution of ventilation ${ }^{8}$ and is reduced primarily by airflow limitation in patients with emphysema. ${ }^{9}$ Spectral changes distinguishing the asthma patients from healthy subjects have been described. ${ }^{3-510}$ In addition, the frequency content of breath sounds was found to be dependent on the degree of bronchial obstruction during challenge tests, even in the absence of wheezing sounds. ${ }^{11-13}$

Standards for the diagnosis of obstructive pulmonary diseases are available. ${ }^{14}$ In clinical and functional patterns there is a considerable overlap between asthma and chronic obstructive pulmonary disease (COPD), although the morphological and structural changes in the bronchi and lung parenchyma, as well as the site of these changes in the bronchial tree, are partly different. ${ }^{1516}$ The different structural and morphological changes in COPD and asthma might be reflected in breath sounds.

At present there are few data available on the spectral characteristics of breath sounds in COPD, and no comparative data on the breath sounds of patients with asthma and COPD. The aim of the present study was to investigate the spectral characteristics of patients with COPD and stable asthma with significant airways obstruction under standardised conditions, and to compare them with those of sex and age matched control patients with healthy lungs.

\section{Methods}

PATIENTS

Thirty eight male outpatients referred to the Lung Function Laboratory at Helsinki University Central Hospital were selected for the study. Their anthropometric and lung function data are presented in table 1 .

Seventeen patients had stable chronic obstructive pulmonary disease diagnosed previously according to ATS criteria ${ }^{14}$; all were heavy current or ex-smokers. The degree of obstruction assessed by spirometric tests ranged from mild to very severe. None of the 
Table 1 Median (range) anthropometric and lung function data of the patients with asthma, chronic obstructive pulmonary disease (COPD) and controls

\begin{tabular}{|c|c|c|c|}
\hline & $\begin{array}{l}\text { Asthma } \\
(n=10)\end{array}$ & $\begin{array}{l}C O P D \\
(n=17)\end{array}$ & $\begin{array}{l}\text { Controls } \\
(n=11)\end{array}$ \\
\hline $\begin{array}{l}\text { Age (years) } \\
\text { Height }(\mathrm{cm}) \\
\text { Weight }(\mathrm{kg}) \text { FEV }_{1}(1) \\
\text { FEV }_{1}\left(\% \text { predicted }^{17}\right) \\
\text { TLCo }\left(\% \text { predicted }^{17}\right) \\
\text { Kco }\left(\% \text { predicted }^{17}\right)\end{array}$ & $\begin{array}{l}50(26-72) \\
176(164-180) \\
76(58-99) \\
2 \cdot 59(1 \cdot 02-3 \cdot 61)^{*} \\
67 \cdot 5(31-79)^{*}\end{array}$ & $\begin{array}{l}58(38-73) \\
172(166-185) \\
76(57-97) \\
1 \cdot 26(0 \cdot 59-3 \cdot 20)^{*} \\
36(16-79)^{*} \\
57(35-93) \\
67(40-105)\end{array}$ & $\begin{array}{l}50(44-66) \\
174(165-176) \\
82(67-87) \\
3 \cdot 55(3 \cdot 26-5 \cdot 05) \\
95(85-124)\end{array}$ \\
\hline
\end{tabular}

$\mathrm{FEV}_{1}=$ forced expiratory volume in one second; $\mathrm{TLCO}=$ carbon monoxide lung transfer factor; $\mathrm{KCO}=$ carbon monoxide transfer coefficient.

${ }_{*}^{*} \mathrm{p}<0.001$ compared with control patients.

patients had $\alpha_{1}$-antitrypsin deficiency. In 10 patients with COPD the specific diffusing capacity for carbon monoxide $(\mathrm{KCO}=$ diffusing capacity (TLCO) related to alveolar volume) was below that predicted, suggesting the presence of emphysema; radiographically there were no signs of interstitial lung disorders such as pulmonary fibrosis. Eleven of the patients with COPD were on $\beta_{2}$ sympathomimetics, nine were on inhaled or oral corticosteroids, eight were on theophylline, and five were taking inhaled ipratropium bromide. In addition, two patients with COPD were on long acting nitrates for mild coronary heart disease.

Ten patients had stable asthma diagnosed previously according to the ATS criteria ${ }^{14}$ but had no other cardiorespiratory disease. None of the asthmatics had smoked during the previous 10 years. The degree of bronchial obstruction ranged from mild to severe. All asthmatics were on regular $\beta_{2}$ sympathomimetics; in addition, seven patients used inhaled or oral corticosteroids, five theophylline, and two ipratropium bromide, but no other drugs.

Eleven patients with suspected coronary heart disease, referred to the laboratory for routine pulmonary function tests prior to coronary angiography, were selected as controls, matched according to age and sex. None had symptoms or signs of any respiratory disease or heart failure, and their spirometric values and chest radiographs were normal. None were current or ex-smokers. They all had regular medication for coronary disease (eight were on acetylsalicylic acid, nine were on long acting nitrates, seven $\beta$ adrenergic blocking agents, and three nifedipine), but not for any respiratory disorder or for cardiac insufficiency; one control patient used digoxin for episodic supraventricular tachyarrhythmia.

No patient in any group had suffered from a respiratory infection during the two weeks preceding the test, and the patients had used their medication regularly when entering into the study.

Informed consent was obtained from every patient and the study was approved by the ethics committee of the Department of Pulmonary Diseases at Helsinki University Central Hospital.

STUDY DESIGN

Spirometric tests were performed on every patient 10-15 minutes before recording of breath sounds. A rolling seal flow volume spirometer (CPI 220, Cardiopulmonary Instruments, Houston, Texas, USA) connected to a microcomputer system (Medikro 202, Medikro Oy, Kuopio, Finland) was used to measure $\mathrm{FEV}_{1}$ and for assessment of the degree of ventilatory impairment; the highest value of at least three successive determinations was recorded. The TLCo and Kco, corrected according to the actual blood haemoglobin concentration, were determined from the patients with COPD by the single breath method ${ }^{18}$ (Masterlab, Erich Jaeger GmbH, Würzburg, Germany). The mean value of two determinations was recorded.

Breath sounds were recorded simultaneously from the chest wall and the trachea. The method has been described earlier. ${ }^{613}$ For recording of lung sounds an air-coupled condenser microphone (B\&K 4134; sensitivity $12.5 \mathrm{mV} / \mathrm{Pa}$; free field frequency response $3-20000 \mathrm{~Hz}(-3 \mathrm{~dB}))$ was attached with a rubber belt to the patient's chest at the right lower lobe area, approximately $10 \mathrm{~cm}$ below the margin of the scapula and $15 \mathrm{~cm}$ to the right of the spine. The exact location was chosen by ordinary auscultation based on sufficient sound intensity. The microphone was encased in the cup of a hearing shelter to reduce ambient noise. The diameter and depth of the slightly conical coupling cavity were 17 and $5 \mathrm{~mm}$, respectively. A small piezoelectric contact sensor (PPG Sensor no. 102, Technion, Haifa, Israel; sensitivity $8 \mathrm{mV} / \mathrm{Pa}$; free field frequency response essentially flat $( \pm 3 \mathrm{~dB})$ within $100-1500 \mathrm{~Hz}$ ) was hand held at the trachea on the right side of the cricothyroid cartilage. The sound signals were prefiltered with a third order high pass filter with a cut off frequency of $50 \mathrm{~Hz}(18 \mathrm{~dB} / \mathrm{oct})$ to prevent saturation of the amplifier by low frequency noise. The amplifier (B\&K 2619) had a flat $( \pm 0.5 \mathrm{~dB})$ frequency response curve over $20-20000 \mathrm{~Hz}$.

During breath sound recording the flow at the mouth was monitored with a pneumotachograph (Medikro MF S202, Medikro Oy, Kuopio, Finland) with a zero centre analogue monitor in front of the patient. Each patient wore a noseclip and was asked to keep his peak inspiratory and expiratory flows at a target level of $1.25 \mathrm{l} / \mathrm{s}$; all patients were carefully trained in the technique before the recording.

The sound and flow signals were recorded with an eight channel DAT recorder (Teac RD-111T, frequency response DC $-5 \mathrm{kHz} \pm 0.5 \mathrm{~dB})$. The analogue output signal 
of the recorder was digitised in a data acquisition and control unit (HP 3852A) with 13-bit analogue-to-digital conversion; the sampling rate was $12 \mathrm{kHz}$ for sound and $100 \mathrm{~Hz}$ for the airflow. A Bessel type low pass filter with a cut off frequency of $4 \mathrm{kHz}(24 \mathrm{~dB} / \mathrm{oct})$ was used to prevent aliasing. The data from the flow and sound signals were stored on a magneto-optical disk of a Unix work station (HP 9000/330C). The sound signal was digitally high pass filtered (Kaiser-FIR) with a cut off frequency of $100 \mathrm{~Hz}(24 \mathrm{~dB} / \mathrm{oct})$.

The flow and sound signals were subsequently analysed on a computer by using custom made software based on Labview tool package. For the spectral analysis of sound signals 2048-point fast Fourier transforms were performed with $50 \%$ overlapping of adjacent Hanning data windows; the spectra were averaged over 8-10 consecutive breathing cycles by the method of Welch. ${ }^{19}$ The effective frequency resolution of the spectral estimates was approximately $5 \mathrm{~Hz}$. Only sound samples of inspiratory sounds that occurred at flows from $1.0 \mathrm{l} / \mathrm{s}$ to target flow $(1.25 \mathrm{l} / \mathrm{s})$ were used for calculation of averaged spectra. The average power spectra of background noise were similarly estimated from sound samples within a flow gate of $0 \pm 0.1 \mathrm{l} / \mathrm{s}$. These spectra were used to evaluate the signal-to-noise ratio of the recordings. Expiratory sounds were not analysed because the breathing pattern could not be standardised among the patient groups due to expiratory flow limitation in some patients with asthma or COPD.

The upper frequency limits for the second quartile $\left(\mathrm{F}_{50}\right.$, the median frequency) and the third $\left(\mathrm{F}_{75}\right)$ quartile of the spectral power were calculated within a measuring band of $100-2000 \mathrm{~Hz}$ on the linear amplitude scale. The frequency of maximum intensity (Fmax) and the lung sound amplitude in terms of the root mean square (RMS) value of the total spectral power were also determined within the same measuring band.

The presence or absence of wheezes in the sound samples was evaluated by visual examination of the sound signal in time-expanded waveform analysis $(1000 \mathrm{~mm} / \mathrm{s})$ and in fre- quency domain (FFT). The criteria for a wheeze were the presence of a sinusoidal waveform with a duration of more than $200 \mathrm{~ms}$ or an intensity peak distinctly separated from its surrounding intensity in the FFT spectrum. Within the inspiratory flow gate chosen for analysis $(1 \cdot 0-1 \cdot 25 \mathrm{l} / \mathrm{s})$ none of the patients was found to wheeze.

\section{DATA ANALYSIS}

The breath sound variables between the study groups were compared by the non-paired $t$ test with the $\mathrm{p}$ values being adjusted for multiple comparison according to the Bonferroni equation $\left(\mathrm{p}^{*}=\mathrm{pK} ; \mathrm{K}=\mathrm{k}(\mathrm{k}-1) / 2\right.$ where $\mathrm{p}^{*}$ is the adjusted value of $p$ and $k$ is the number of groups). ${ }^{20}$ For correlative analysis within the groups, Pearson's correlation coefficient was calculated. A p value of less than 0.05 was considered significant.

\section{Results}

Examples of phonopneumograms and averaged inspiratory spectra recorded at the chest in patients with asthma, COPD, and normal lungs are illustrated in fig 1 . The spectra are calculated from sound samples at an airflow gate of $1 \cdot 0-1 \cdot 25 \mathrm{l} / \mathrm{s}$. In each spectrum plotted on a $\log -\log$ scale the $F_{50}$ is indicated and the background spectra (sound at $0 \pm 0 \cdot 1 \mathrm{l} / \mathrm{s}$ flow) presented. The expiratory flow profiles differed between the patients with different diagnoses as shown in fig 1, but inspiratory flow profiles appeared quite similar. The spectra in fig 1 are smooth in shape without irregularities or peaks that could indicate wheezing sounds. The spectrum of the asthma patient is broader in shape, indicating increased sound energy at a frequency range of $200-1000 \mathrm{~Hz}$; consequently, the $F_{50}$ is increased compared with that of the control patient. In the patient with COPD the attenuation of the sound power is more gradual over the whole frequency range, but the global frequency distribution in terms of $F_{50}$ is not significantly different from that of the control patient. In the whole group of patients with COPD we did not find any characteristic spec-
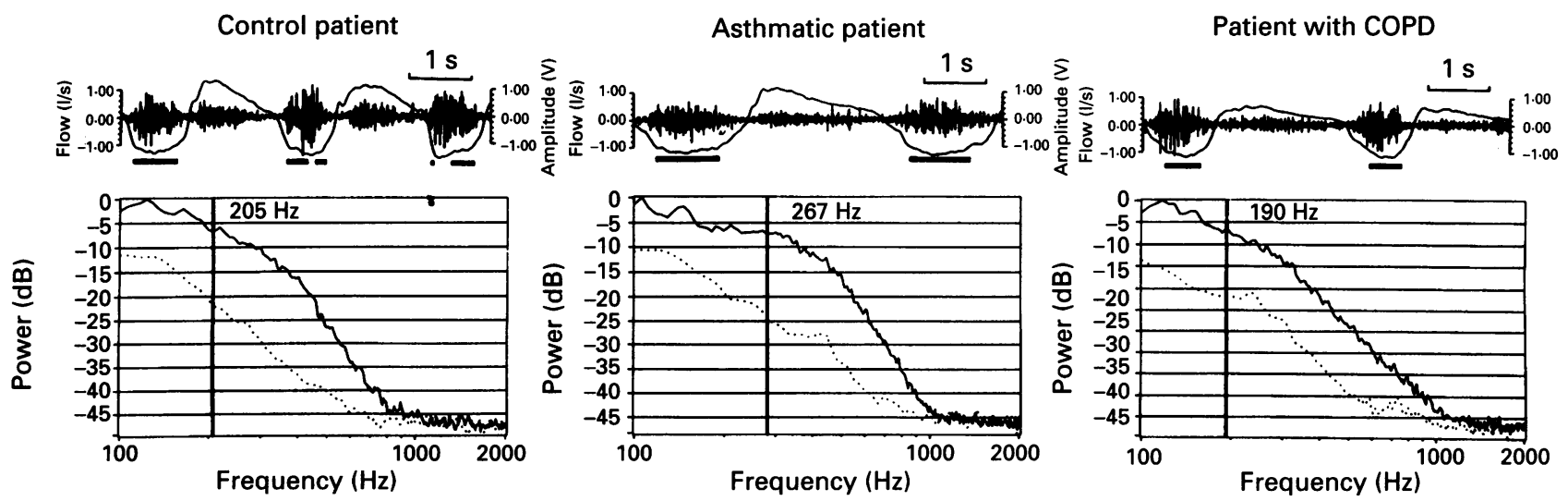

Figure 1 Examples of phonopneumograms and flow standardised inspiratory spectra recorded at the chest in a control patient and patients with asthma and COPD. Only sound samples at a flow gate of $1 \cdot 0-1 \cdot 25 \mathrm{l} / \mathrm{s}$ (indicated as lines under the flow curve) were used for calculation of the averaged spectra. In each spectrum the median frequency (solid vertical line) is indicated and the background (sound at $0 \pm 0 \cdot 1 \mathrm{lls}$ flow) spectra presented (dotted line). 


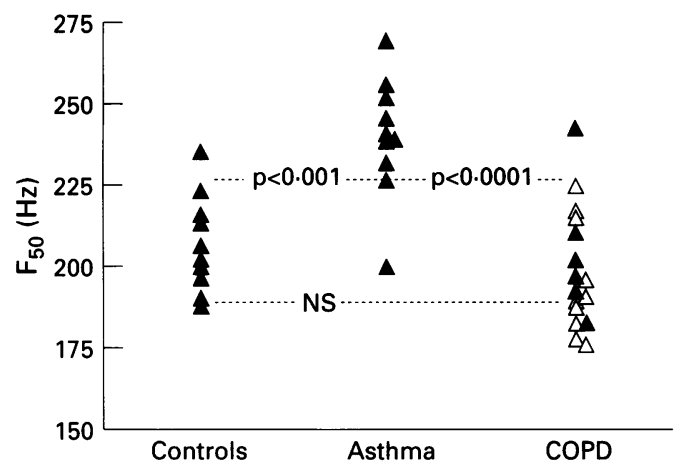

Figure 2 Distribution of inspiratory median frequency $\left(F_{50}\right)$ of breath sounds recorded at the chest in patients with asthma, COPD, and healthy lungs (controls). In the COPD group open triangles refer to patients with reduced specific diffusing capacity.

tral pattern different from that of control patients

The values of the breath sound parameters in the three patient groups are presented in table 2 . The inspiratory $F_{50}$ and $F_{75}$ recorded at the chest wall were significantly higher $(p$ $<0.001$ and $p<0.01$, respectively) in the asthmatic than in the control patients. The $\mathrm{F}_{50}$ of the patients with COPD did not differ significantly from that of the control patients but was significantly lower than that of the asthmatics ( $p$ $<0.0001$ ). The distribution of $\mathrm{F}_{50}$ recorded at the chest in each of the three patient groups is illustrated in fig 2 . The spectral parameters recorded at the trachea did not differ significantly among the patient groups.

In patients with asthma the $F_{50}$ of breath sound spectra recorded at the trachea was related to $\mathrm{FEV}_{1}(r=-0.77, \mathrm{p}<0.01)$ and a similar but not statistically significant relationship was recorded at the chest $(r=-0.50, \mathrm{p}<0 \cdot 10)$; patients with severe obstruction had the highest $\mathrm{F}_{50}$ values. In patients with COPD and in controls the $\mathrm{F}_{50}$ was not significantly related to $\mathrm{FEV}_{1}$ either at the chest or at the trachea. In patients with COPD the inspiratory $\mathrm{F}_{50}$ seemed to be weakly related to $\mathrm{KcO}$ so that those patients with reduced $\mathrm{Kco}$ had lower $\mathrm{F}_{50}$ values; however, the correlation was not statistically significant $(r=0 \cdot 43, \mathrm{p}<0 \cdot 10)$.

The total spectral power recorded at the chest in terms of RMS was higher in asthmatics than in patients with COPD. Otherwise, the

Table 2 Mean (SD) total spectral power (RMS), frequency of maximum intensity (Fmax), and upper frequency limits for the second $\left(F_{50}\right)$ and the third $\left(F_{75}\right)$ quartile of the power spectra of flow standardised inspiratory breath sounds recorded at the chest wall and at the trachea in patients with asthma and chronic obstructive pulmonary disease (COPD) and in controls

\begin{tabular}{llll}
\hline & $\begin{array}{l}\text { Asthmatics } \\
(n=10)\end{array}$ & $\begin{array}{l}\text { COPD } \\
(n=17)\end{array}$ & $\begin{array}{l}\text { Controls } \\
(n=11)\end{array}$ \\
\hline Chest & & & \\
RMS (dB) & $67 \cdot 1(4 \cdot 5)$ & $63 \cdot 5(4 \cdot 4)$ & $64 \cdot 4(4 \cdot 4)$ \\
Fmax (Hz) & $129(33)$ & $113(17)$ & $117(18)$ \\
$\mathrm{F}_{50}(\mathrm{~Hz})$ & $239(19)^{* *}$ & $201(21)$ & $206(14)$ \\
$\mathrm{F}_{75}(\mathrm{~Hz})$ & $370(48)^{*}$ & $321(51)$ & $301(33)$ \\
Trachea & & & \\
RMS (dB) & $84 \cdot 3(3 \cdot 7)$ & $82 \cdot 6(3 \cdot 1)$ & $83 \cdot 1(3 \cdot 7)$ \\
Fmax (Hz) & $325(433)$ & $228(340)$ & $154(157)$ \\
$\mathrm{F}_{50}(\mathrm{~Hz})$ & $856(231)$ & $753(177)$ & $766(178)$ \\
$\mathrm{F}_{75}(\mathrm{~Hz})$ & $1298(203)$ & $1239(186)$ & $1323(192)$ \\
\hline
\end{tabular}

${ }^{*} \mathrm{p}<0.01,{ }^{* *} \mathrm{p}<0.001$ compared with control patients ( $\mathrm{p}$ adjusted for multiple comparisons)
RMS was not significantly different among the patient groups. The Fmax varied between individuals, especially at the trachea, and no significant differences between the study groups were found.

\section{Discussion}

The results of this study indicate that the frequency content of breath sounds in asthmatic patients with airways obstruction differs markedly from that of patients with COPD and those with normal lungs. This observation may reflect different pathophysiological mechanisms of lung sound generation or transmission between asthma and COPD, and this may provide new tools for the differential diagnosis of obstructive pulmonary diseases. Breath sounds detected at the chest wall and at the trachea are, to some extent, influenced by the physical dimensions of the airways and structure of the surrounding lung tissue..$^{2122}$ In COPD histological changes of peripheral airways disease and emphysema can be found with both inflammation of the terminal and respiratory bronchioles and fibrosis of the airways walls. In asthma the bronchial mucosa is infiltrated with inflammatory cells, together with shedding of the epithelium and smooth muscle hypertrophy. The type of structural change and its site in the bronchi and lung parenchyma differ between COPD and asthma, ${ }^{151623}$ and this may account for the differences in the frequency content of breath sounds.

The patients in this study were carefully selected to meet the ATS criteria for asthma and COPD,${ }^{14}$ but had no other cardiorespiratory disease; only non-smokers were included in the asthma group to ensure a diagnosis of pure asthma. Since peripheral airways disease and emphysema often coexist in COPD and are sometimes clinically difficult to distinguish, the patients with COPD were treated as a single group. It may be argued that the control patients did not represent a true healthy population. However, they were non-smokers with no respiratory disorders or heart failure which might alter the breath sounds. Most of these control patients used $\beta$ adrenergic blocking agents which are known to alter the bronchial tone in asthmatics; however, in patients with no history of asthma $\beta$ adrenergic blockade should have no significant effects on the bronchi. ${ }^{24}$ In addition, the findings of flow-volume spirometry, chest radiography, and pulmonary auscultation were normal in all control patients so we regarded their lungs as normal. Breath sound spectra have been found to depend on age $^{25}$ and on body height ${ }^{26}$; however, the present study groups did not differ markedly with respect to sex, age, or body size. The average severity of ventilatory impairment in asthma was lower than in COPD; however, with even more severe bronchial obstruction one would expect to find similar or higher $F_{50}$ values in patients with COPD than in asthmatics. Since the $F_{50}$ values in COPD were, in fact, lower than in asthmatics, we believe that these changes are 
not explained by differences in the severity of airways obstruction.

High quality equipment was used for recording of breath sounds. ${ }^{6}$ Different microphones were used at the trachea and at the chest for anatomical reasons so the sound spectra cannot be critically compared between the two locations. It should be noted that the frequency characteristics of the microphones, ${ }^{27}$ and specifically the dimensions of the air coupling cavity, ${ }^{28}$ have been shown to modify the shape of lung sound spectra. With the present condenser microphone of $5 \mathrm{~mm}$ cavity depth some high frequency components may therefore have been attenuated in the chest wall recordings. However, the same microphones were used for all patients and their characteristics cannot therefore explain the differences among the patient groups.

The frequency spectra were averaged to minimise the effect of random noise. A cutoff frequency level of $100 \mathrm{~Hz}$ was chosen to eliminate the muscular ${ }^{29}$ and cardiovascular ${ }^{30}$ sounds from the respiratory sound analysis. Calculation of background noise enabled us to evaluate the signal-to-noise ratio of the sound. The background spectra were not subtracted from the analysed breath sound spectra because relevant information may have been lost for two reasons. Firstly, the background spectra were derived under dynamic conditions (during breathing with flows up to $\pm 0.01 \mathrm{l} / \mathrm{s}$ ) and inevitably contained a small contribution of lung sounds. Secondly, in some individuals there were insufficient data points to create an accurate estimate from the background spectrum (even if the level of signal-to-noise ratio could be estimated). We believe that, if subtraction of background noise is preferred, sound during breath holding should be used. ${ }^{31}$ Our preference to use background noise at zero flow as quality control is the same as that of Pasterkamp et al. ${ }^{27}$ The same recording room and conditions were used for every patient so the ambient noise during recordings did not vary significantly from patient to patient.

The flow at the mouth was carefully controlled during lung sound recordings so that inspiratory tidal peak flow and pattern were similar in the patient groups. The spectral shape has been shown to be quite stable at high flow rates above $1.01 / \mathrm{s},{ }^{32} 33$ but to be dependent on flow at low flow rates. ${ }^{310}$ Flow gated sampling of respiratory sounds was originally described by O'Donnel and Kraman. ${ }^{34}$ In this study we used only sound samples of a defined inspiratory flow gate $(1 \cdot 0-1 \cdot 25 \mathrm{l} / \mathrm{s})$ for the spectral analysis, ensuring that breath sound spectra among the patient groups were derived from exactly the same flows. Differences in airflow or breathing pattern cannot therefore explain the differences in frequency distribution of lung sounds among the patient groups. Since only inspiratory breath sounds were analysed, expiratory flow limitation occurring in some patients with COPD did not influence the results. In contrast, clear differences in expiratory flow patterns did not allow valid comparison of expiratory breath sounds so the latter were not included in the study.
Compared with control and COPD patients, the different frequency content of breath sounds in the asthmatics was reflected in changes in $\mathrm{F}_{50}, \mathrm{~F}_{75}$, and the total spectral power. The presence of adventitious lung sounds such as wheezes and crackles could change these spectral indices, but there were no wheezing sounds during the inspiratory sound samples selected for analysis. Crackling sounds were not counted, but on visual examination appeared to be rare in our patients with COPD. Furthermore, the averaging method used is relatively insensitive to non-stationary changes of breath sounds such as crackles. It is therefore unlikely that adventitious breath sounds would have markedly contributed to the changes observed in averaged frequency spectra.

It may be argued that the present parameters chosen to characterise the breath sound spectra (RMS, Fmax, and the quartiles) are not sufficient as they do not provide specific information about the pattern of the spectral curve. However, they have been widely used in clinical studies of breath sounds in asthma $a^{10113}$ and, in particular, the quartiles give an estimate of the global frequency distribution of breath sounds, the primary interest of our study.

Normal tracheal sounds are characterised by a broad spectrum of frequencies with a sharp decrease of power above $800-1600 \mathrm{~Hz}$. Normal vesicular lung sounds in the chest contain a spectrum of frequencies up to approximately $500 \mathrm{~Hz}$; the frequency components of lung sounds above this are effectively filtered by the lung tissue and the chest wall. ${ }^{35}$ In normal men inspiratory sounds containing substantial power relative to background noise up to $2000 \mathrm{~Hz}$ have been reported when high airflow rates are used (up to $2.5 \mathrm{l} / \mathrm{s}$ ). ${ }^{27}$ In the present study the usual frequency range at which the background noise was reached in chest recordings was $1 \cdot 0-1 \cdot 2 \mathrm{kHz}$. However, the target flow was set at a relatively low level $(1 \cdot 25 \mathrm{l} / \mathrm{s})$ due to the reduced ventilatory function in patients with asthma and COPD.

In previous studies the frequency spectra of asthmatics were found to differ from those of healthy controls during episodes of wheezing. ${ }^{3-5}$ In patients with acute asthma the proportion of the respiratory cycle occupied by wheezing was found to correlate with $\mathrm{FEV}_{1}{ }^{36}$ In previous studies on asthmatics during challenge tests the frequency distribution of breath sounds was shown to be related to the degree of bronchial obstruction, with higher $F_{50}$ values being found during bronchial obstruction ${ }^{11-13}$ and also in the absence of wheezing sounds. Schreur et $a l^{10}$ recently found similar changes in frequency distribution of lung sounds in asthma patients even with normal lung function. The present finding that sound energy is shifted towards higher frequencies in asthmatics compared with controls is in accordance with previously reported studies.

In the previous literature there are only few data available about the spectral characteristics of breath sounds in COPD. Gavriely et $a l^{37}$ used lung sound analysis for respiratory health screening and found that 30 out of 62 subjects with clinical COPD had abnormal breath 
sound spectra. They used regression analysis of the spectral curve to define abnormality, which is a particularly useful method to detect curve irregularities caused by wheezing sounds. The quartile frequencies were not used in this study, and the patterns of abnormalities in COPD were not compared with those in asthma. In our study flow standardised inspiratory breath sounds that did not contain wheezes did not differ between COPD and control patients, but in asthmatic patients significantly higher RMS, $F_{50}$ and $F_{75}$ values were found than in patients with COPD. The spectral shapes did not differ visually between patients with COPD and control patients, and the frequency range that contained breath sounds with substantial power relative to background spectra was about the same in the two groups. However, the frequency content of inspiratory vesicular lung sounds in patients with COPD seemed to be affected by the presence of emphysema, patients with reduced Kco having lower $F_{50}$ values.

It has been shown that the auscultatory finding of damped breath sounds in patients with emphysema is predominantly due to airflow limitation, and that at standardised airflows the breath sound intensity is similar in patients with emphysema and healthy controls. ${ }^{9}$ The RMS values in the present study were calculated from the power spectrum and are not therefore equivalent to breath sound intensity. However, our finding that the total spectral power did not differ between the COPD patients and controls was consistent with the previous literature. During episodes of normal lung function in asthmatic patients Schreur et $a l^{10}$ found that, at a given airflow, expiratory sounds were lower in intensity than those of the healthy controls. In contrast, they did not find any difference in inspiratory sound intensity between their study groups as we did between the control patients and asthmatic patients with airways obstruction.

The generally accepted view is that vesicular lung sounds are generated predominantly by turbulence in the larger airways (main, lobar, and segmental bronchi) and transmitted from these locations to the chest wall. ${ }^{21}$ The calculations from gas density, viscosity, and velocity predict laminar and, presumably, soundless flow in more peripheral airways. However, there is experimental evidence to show that at least the inspiratory portion of vesicular lung sounds is produced locally in small airways by non-turbulent mechanisms. ${ }^{38}$ In asthmatic patients the increase in frequency content of breath sounds during bronchoconstriction can be explained by the local increase of flow velocity through the narrowed bronchi which results in increased kinetic energy and turbulence, characterised by a higher pitch sound. ${ }^{39}$ According to the model of unstable vortices by Hardin and Patterson ${ }^{40}$ the sound frequency produced is inversely related to the diameter of the airways at a given mass flow; higher sound frequencies are thus expected to be found in asthmatics with narrowed airways. The present finding that the $F_{50}$ is significantly correlated with $\mathrm{FEV}_{1}$ in asthmatic patients also indicates the close relationship between frequency distribution of breath sounds and narrowing of the airways.

The present results indicate that, in patients with COPD, the vesicular lung sounds are either unchanged in spite of airway narrowing or some other mechanisms are involved that counteract the effect of increased turbulence in the bronchi. The sound generation in asthma and COPD may be different since the structural changes in the bronchi in patients with COPD are situated more peripherally in small airways where turbulence does not occur in normal conditions. The trend between $\mathrm{KcO}$ and $\mathrm{F}_{50}$ of inspiratory lung sounds in COPD suggests that the sound is affected by the degree of emphysema. The lung parenchyma and the chest wall act like a band pass filter. The average density of lung tissue is reduced in emphysema due to tissue destruction; this should affect the transmission of lung sounds from their source to the chest wall ${ }^{22}$ and accentuate the low pass filtration effect of the thorax. Consequently, lower frequency lung sounds would be detected at the chest, a possible explanation for the difference between asthma and COPD.

Breath sounds heard at the trachea are presumably generated in the very central airways by turbulent mechanisms, and the larynx may also contribute to the sound production by acting as a nozzle, creating a jet of air. ${ }^{21}$ The correlation between $\mathrm{FEV}_{1}$ and $\mathrm{F}_{50}$ in asthmatic patients may relate to increased turbulence in central airways, probably caused by airway narrowing. The frequency distribution of tracheal sounds has high intersubject variation which relates to individual resonant frequencies with accentuated intensities. Thus, significant differences between the patient groups could not be found for tracheal sounds.

The finding that breath sound spectral patterns in patients with COPD and asthma differ significantly may have also clinical implications, since it provides new tools for differential diagnosis of obstructive lung disorders. In the present study, however, the $F_{50}$ values in asthma and in COPD partly overlapped, and the specificity of a given $F_{50}$ value is probably not very high. Further studies may reveal other spectral estimators that could be used to separate the groups more effectively.

In conclusion, the present findings indicate that the frequency spectra of breath sounds in patients with stable asthma, but not in patients with COPD, differ significantly from those in patients with healthy lungs. It is postulated that the structural changes in the bronchi and in the surrounding lung tissue in COPD and asthma result in different breath sound generation or transmission. Breath sound spectral analysis may provide a new non-invasive method for differential diagnosis of bronchial obstruction.

This study was supported by the Ida Montin Foundation, Finland. The authors are grateful to Erkki Paajanen and Kari Kallio for their technical collaboration.

1 Murphy RLH, Sorensen K. Chest auscultation in the diagnosis of pulmonary asbestosis. $\mathcal{F}$ Occup Med 1973;15 272-6.

2 Murphy RLH, Holford SK, Knowler WC. Visual lungsound characterization by time-expanded wave-form analysis. N Engl ₹ Med 1977;296:968-71. 
3 Charbonneau G, Racineux JL, Sudraud M, Tuchais E. An accurate recording system and its use in breath sounds spectral analysis. $\mathcal{f}$ Appl Physiol 1983;55:1120-7.

4 Gavriely N, Palti Y, Alroy G, Grotberg JB. Measurement and theory of wheezing breath sounds. F Appl Physiol 1984;57:481-92.

5 Pasterkamp H, Carson C, Daien D, Oh Y. Digital respirosonography. New images of lung sounds. Chest 1989; 96:1405-12.

6 Rosqvist T, Paajanen E, Kallio K, Rajala H-M, Katila T, Malmberg LP. A toolkit for lung sound analysis. Med Bio Eng Comput 1995;33:190-5.

7 Forgacs P, Nathoo AR, Richardson HD. Breath sounds. Thorax 1971;26:288-95.

8 Ploysongsang Y, Pare JAP, Macklem PT. Correlation of regional breath sounds with regional ventilation in emphysema. Am Rev Respir Dis 1982;126:526-9.

9 Schreur HJW, Sterk PJ, Vandershoot J, van Klink HCJ, van Vollenhoven E, Dijkman JH. Lung sound intensity in patients with emphysema and in normal subjects at standardized airflows. Thorax 1992;47:674-9.

10 Schreur HJW, Vandershoot J, Zwinderman AH, Dijkman $\mathrm{JH}$, Sterk PJ. Abnormal lung sounds in patients with asthma during episodes with normal lung function. Chest 1994;106:91-9.

11 Andersson K, Aitken S, Carter R, MacLeod JES, Moran F. Variation of breath sound and airway caliber induced by histamine challenge. Am Rev Respir Dis 1990;141: 1147-50.

12 Spence DPS, Bentley S, Evans DH, Morgan MDL. Effect of methacholine induced bronchoconstriction on the spectral characteristics of breath sounds in asthma. Thorax 1992; 47:680-3.

13 Malmberg LP, Sovijärvi ARA, Paajanen E, Piirilä P, Haahtela $T$, Katila $T$. Changes in frequency spectra of breath sounds during histamine challenge test in adult asthmatics and healthy controls. Chest 1994;105:122-31.

14 American Thoracic Society. Standards for the diagnosis and care of patients with chronic obstructive pulmonary disease (COPD) and asthma. Am Rev Respir Dis 1987;136:225-44.

15 McDowell EM, Beals TF. Biopsy pathology of the bronchi Philadelphia: W B Saunders, 1987.

16 Laitinen LA, Laitinen A. Pathology and cytology of asthma. In: Clark TJH, Godfrey S, Lee TH, eds. Asthma. London: Chapman \& Hall, 1992:232-53.

17 Viljanen AA, Halttunen PK, Kreu K-E, Viljanen BC. Reference values for spirometric, pulmonary diffusing capacity and body plethysmographic studies. Scand $\mathcal{f}$ Clin Invest 1982;42(Suppl 159):1-50.

18 Cotes JE, Chinn DJ, Quanjer PH, Roca J, Yernault JC. Standardization of the measurement of transfer facto (diffusing capacity). Eur Respir f 1993;6(Suppl):41-52.
19 Welch PD. The use of fast Fourier transform for the estimation of power spectra. IEEE Trans Audio Electroacoust timation of power spe

20 Snedecor GW, Cochran WG. Statistical methods. 7th edn Ames: The Iowa State University Press, 1982.

21 Olson DE, Hammersley JR. Mechanisms of lung sound generation. Semin Respir Med 1985;6:171-9.

22 Rice DA. Transmission of lung sounds. Semin Respir Med 1985;6:166-70.

23 Laennec RTH. $A$ treatise on diseases of the chest. Translated from the French by John Forbes. London: T. and C. Underwood, 1821

24 Richardson PS, Sterling GM. Effects of beta-adrenergic receptor blockade on airway conductance and lung volum in normal and asthmatic subjects. $B M \mathcal{F} 1969 ; 3: 580-4$.

25 Hidalgo HA, Wegmann MJ, Waring WW. Frequency spectra of normal breath sounds in childhood. Chest 1991;100: 999-1002.

26 Sanchez I, Pasterkamp H. Tracheal sound spectra depend on body height. Am Rev Respir Dis 1993;148:1083-7.

27 Pasterkamp H, Kraman SS, DeFrain PD, Wodicka GR. Measurement of respiratory signals. Comparison of sensors. Chest 1993;104:1518-25.

28 Wodicka GR, Kraman SS, Zenk GM, Pasterkamp $H$ Measurement of respiratory acoustic signals. Effect of microphone air cavity depth. Chest 1994;106:1140-4.

29 Kraman SS. Does the vesicular breath sound come only from the lungs? Am Rev Respir Dis 1983;128:622-6.

30 Pasterkamp H, Fenton R, Tal A, Chernick V. Interference of cardiovascular sounds with phonopneumography in of cardiovascular sounds with phonopneumo

31 Gavriely N, Cugell D. Breath sounds methodology. Boca Raton, Florida: CRC Press Inc., 1995.

32 Kraman SS. Effects of lung volume and airflow on the frequency spectrum of vesicular lung sounds. Respir Physiol frequency spectr

33 Mussel MJ, Nakazano Y, Miyamoto Y. Effect of air flow and flow transducer on tracheal breath sounds. Med Biol Eng Comput 1990;28:550-4.

34 O'Donnel DM, Kraman S. Vesicular lung sound amplitude mapping by automated flow-gated phonopneumography. f Appl Physiol 1982;53:603-9.

35 Gavriely N, Palti Y, Alroy G. Spectral characteristics of normal breath sounds. $\mathcal{F}$ Appl Physiol 1981;50:307-14

36 Baughman RP, Loudon RG. Quantitation of wheezing in acute asthma. Chest 1984;86:718-22.

37 Gavriely N, Nissan M, Cugell DW, Rubin A-HE. Respiratory health screening using pulmonary function test and lung sound analysis. Eur Respir ₹ 1994;7:35-42.

38 Kraman SS. Determination of the site of production of respiratory sounds by subtraction phonopneumography. Am Rev Respir Dis 1980;122:303-9.

39 Olson DE, Snyder B. Origin of central airway breath sounds. Am Rev Respir Dis 1983;127:A268.

40 Hardin JC, Patterson JL. Monitoring the state of the human airways by analysis of respiratory sound. Acta Astronaut 1979;6:1137-51. 